\title{
Reply to the Commentary by Naglieri and Das on the Factor Structure of a Battery of PASS Cognitive Tasks
}

\author{
John H. Kranzler \\ University of Florida \\ Li-Jen Weng \\ National Taiwan University
}

\begin{abstract}
Much of the commentary by Naglieri and Das (this issue) results from misinterpretation of the aims and conclusions of our study (Kranzler \& Weng, this issue). Contrary to their interpretation, we did not attempt to develop a new theory of the structure of human intelligence; nor did we generalize from our results to all future batteries of PASS tests. Consequently, our conclusions and interpretations, which pertain only to the factor structure of the battery of tests presented in Naglieri, Das, Stevens, and Ledbetter (Journal of School Psychology, 29, 1-17, 1991), cannot be refuted by their comments. Because the battery of PASS tests in the more recent Das-Naglieri: Cognitive Assessment System Standardization Edition (J. P. Das \& J. A. Naglieri, Chicago: Riverside, 1993) reportedly differs from the test battery that we reanalyzed, attempts at replication of our results with children in Grades 5-12 are needed.
\end{abstract}

Keywords: Cognitive processes; Intelligence; Intelligence measures; Psychometric $g$.

In their commentary, Naglieri and Das (this issue) are critical of many aspects of our study (Kranzler \& Weng, this issue). Much of their critique, however, results from misinterpretation of our aims and conclusions. In this reply, we respond to the major issues raised by Naglieri and Das concerning the factor analysis results of our study. We will not address the much broader and deeper question of the veracity of the PASS theory (for reviews, see Lambert, 1990; Reschly \& Wilson, 1990; Telzrow, 1990), as this is beyond the scope of our original article and this response.

\section{ON THE AIMS OF OUR STUDY}

Naglieri, Das, Stevens, and Ledbetter (1991) used confirmatory factor analysis (LISREL) to examine the factor structure of a battery of tasks hypothesized to measure the constructs of the planning, attention, and simultaneoussuccessive (PASS) processes model of human cognition. After comparing the relative fit provided by the PASS and four alternative models of intelligence,

Received April 21, 1994; final revision received June 8, 1994.

Address correspondence and reprint requests to John H. Kranzler, PhD, 1403 Norman Hall, University of Florida, Gainesville, FL 32611-2053. 
they concluded that the PASS model best described the factor structure of this particular battery of tests. These data notwithstanding, it appeared to us (Kranzler \& Weng, this issue) that Naglieri et al. did not conduct a rigorous test of the superiority of the PASS model because the alternative models they used are easily confutable. Therefore, to further investigate the factor structure of the battery of PASS tests presented in Naglieri et al., we reanalyzed their own data to compare the fit provided by the PASS model and two additional models.

The first of these additional models was hierarchical, with four first-order PASS factors and one second-order general factor (psychometric $g$ ). Hierarchical theories of the structure of mental ability have received considerable support in the literature. For example, Carroll's (1993) recently published book, Human Cognitive Abilities: A Survey of Factor Analytic Studies, in which he reports the results of reanalyses of 467 data sets of human abilities, is a monumental achievement in the field of human intelligence (for reviews, see Brody, 1994; Eysenck, 1994; Sternberg, 1994). According to Carroll, "There is abundant evidence for a factor of general intelligence" (p. 624), as well as for more than 30 first-order factors relevant to the study of cognitive abilities. In Carroll's three-stratum theory of cognitive abilities, psychometric $g$ is at the apex of the factor hierarchy. The hierarchical model we examined is simply an extension of the PASS model to include a second-order $g$. Naglieri and Das suggest that a hierarchical model with a third-order $g$ might have been more appropriate, apparently without considering the difficulties and limitations of hierarchical factor analysis. As Carrol! states,

the major problem is the inevitable consequence of the fact that the number of variables (factors) automatically decreases with the order of analysis. If one starts with what has often been thought of as a large number of original variables (e.g., the 57 variables studied by [L. L.] Thurstone, 1938 [Primary Mental Abilities, Psychometric Monographs, No. 1A], dataset THUR21A), there might be somewhere around, say, seven to fifteen firstorder factors identified, if the study is well designed. But at the second order of analysis, even fifteen factors (as variables) are barely enough to support a highly determined solution. One is fortunate to obtain as many as three second-order factors, and this is the minimum number required to support an analysis for a single factor at the third order. If only two correlated second-order factors are obtained, their loadings on a third-order factor are indeterminate. (Carroll, 1993, pp. 578-579)

The hierarchical model we examined, however, appeared to be a plausible model specifying a single general factor at the highest level of the factor hierarchy, given the relatively small battery of tests reported by Naglieri et al. (1991).

The second additional model we examined was a revised PASS model, with separate Simultaneous and Successive factors and a combined Planning/ 
Attention factor, which we called the (PA)SS model. This model was not suggested by theory, but rather by the perfect correlation between the Planning and Attention factors for the Grades 5-12 group reported in Naglieri et al. A correlation of 1.00 between these two factors indicates that they are indistinguishable. It is important to note that in confirmatory factor analysis "the initial model of the investigator need not be correct or best for the data" (Jöreskog \& Sörbom, 1989, p. 224). Models often must be modified to determine the most parsimonious model with the best fit to the data. This common practice in LISREL is referred to as a "specification search." By examining the fit provided by the (PA)SS model, we simply tested a reasonable hypothesis, based on their results, concerning the latent sources of variance underlying individual differences in the battery of PASS tests in Naglieri et al. We tried to prevent misinterpretation of our intentions by observing that

it is important to note that the aim of this study was not to develop a theory of the structure of mental ability, but to further investigate the factor structure of a battery of tasks hypothesized to measure the PASS constructs. (p. 155)

As a further caveat, we discussed (a) the shortcomings associated with the use of LISREL as an exploratory factor analysis tool and (b) the appropriate role of exploratory and confirmatory factor analysis in the development of theory.

\section{ON THE RESULTS OF OUR STUDY}

Our findings indicated that the original PASS model does not provide the best factorial description of the tests presented in Naglieri et al. (1991). Naglieri and Das (this issue) disagree with this conclusion by emphasizing the nonsignificant differences in chi-squares $\left(\chi^{2}\right)$ among the PASS, (PA)SS, and PASS $+g$ models. They ask, "Should one therefore reject the PASS model on the basis of the absence of difference?" ( $p$. 161, their emphasis). By the very construction of the $\chi^{2}$ test, however, one cannot reject the PASS model when it is the more complex of two models being compared. In the $\chi^{2}$ test in LISREL, the null hypothesis is that the data conform to the simpler of the two models compared. The alternative hypothesis, which one accepts only after rejection of the null hypothesis, is that the data conform to the more complex model. Failure to reject the null hypothesis means that the data do not allow the conclusion that the more complex model is necessary. In the test of the difference in $\chi^{2}$ between the (PA)SS and PASS models, the PASS model is the more complex of the two. The nonsignificant $\chi^{2}$ difference between these models, therefore, does not support the PASS model. Because the (PA)SS model is the simpler of the two models compared in this case, the nonsignificant difference actually supports it, not the PASS model. In addition to the nonsignificant difference 
in $\chi^{2}$ between the PASS and (PA)SS models, every other goodness-of-fit index we examined, excepting the root mean square residual (RMR), indicated that the best fit to the data was provided by the (PA)SS model.

Assessing model fit in LISREL includes more than simply examining the goodness-of-fit indices, however. It also requires examining the factor solution. This involves scrutinizing the parameter estimates, standard errors, correlations of parameter estimates, squared multiple correlations, and coefficients of determination for each model. For the PASS model, examination of the parameter estimates revealed a correlation of +1.06 between the Planning and Attention factors. According to Jöreskog and Sörbom (1989), "Parameter estimates should have the right sign and size. Examples of unreasonable values of parameter estimates are negative variance, correlations which are larger than one in magnitude, [and] covariance or correlation matrices which are not positive definite" (p. 41). In their reply, Naglieri and Das (this issue) state that it is "legitimate" to obtain correlations larger than unity in magnitude in LISREL. Although it is certainly possible by using LISREL to obtain results that are outside the proper parameter space, such results nevertheless indicate that the model is either poorly fitted or overfitted. Therefore, because the correlation of +1.06 between the Planning and Attention factors is outside the proper parameter space, our results indicated that the PASS model provides an improper factor solution for the data reported by Naglieri et al. Of the three models we examined, only the (PA)SS model had an acceptable factor solution.

Naglieri and Das (this issue) also object to our application of the principle of Occam's razor in LISREL modeling. Although we concur that the most parsimonious explanation of any given data set should be preferred over a more complex explanation when all else is equal, we strongly disagree with their contention that "Kranzler and Weng fail to recognize that the comparison is not equal' (p. 163, their emphasis). We do, in fact, recognize that the comparison is not equal. Not only are the goodness-of-fit indices for the (PA)SS model better than those for the other two models, excepting the RMR, but examination of the solution revealed that the PASS and PASS + $g$ models provided improper descriptions of the factor structure for the data reported by Naglieri et al. The PASS, (PA)SS, and PASS $+g$ models, therefore, are not equal. The (PA)SS model provided the best description of the factor structure for their data. It is also important to note that, contrary to the assertion by Naglieri and Das, determination of the best fit to the data in LISREL is based on analysis of empirical evidence, not theory. Theory may suggest a model, but only statistical procedures such as confirmatory factor analysis can test it.

In addition to providing the best fit to the data, the (PA)SS model is also more parsimonious than the other two models. In LISREL, parsimony is determined empirically by comparing the degrees of freedom $(d f)$ of each respective model examined, with greater parsimony corresponding to more 
$d f$. Because the (PA)SS model ( $d f=32)$ has more degrees of freedom than either the PASS $(d f=29)$ or PASS $+g(d f=31)$ models, the (PA)SS model is the most parsimonious. On the basis of these results, we did not argue in favor of the (PA)SS model as the most parsimonious theory of human cognitive functioning, as Naglieri and Das suggest. Rather, we argued in favor of the (PA)SS model as the most parsimonious description of the latent variables underlying individual differences in the battery of tests presented in Naglieri et al. (1991). Hence, our statement that the (PA)SS is the most parsimonious model for the data we reanalyzed is correct.

Incidentally, we are surprised that Naglieri and Das take issue with the size of the data set we reanalyzed. These are, after all, their data. Moreover, Naglieri and Das (this issue) are incorrect in stating that our Tables 4 and 5 are "inconclusive." For the data reported by Naglieri et al., these are the standardized factor loadings and interfactor correlations for the PASS $+g$ and (PA)SS models. In LISREL, the factor solution obtained for one model is not affected by examination of any other model.

\section{ON THE INTERPRETATION OF OUR DATA}

We concluded our study by stating, "As these results do not support the superiority of the PASS model over other conceptualizations of intelligence in describing these data, further refinement of the PASS theory or tests, or perhaps both, appears to be necessary" (Kranzler \& Weng, this issue). Clearly, we did not reject the PASS theory on the basis of a single reanalysis of one data set, as Naglieri and Das (in press) assert. We actually do not know why the PASS model provides an improper factor solution for the battery of tests presented in Naglieri et al. (1991). Although the results of factor analysis cannot be used to reject a neuropsychological theory of brain functioning, confirmatory factor analysis techniques are the only means presently available for testing models of the latent sources of variance underlying individual differences on a battery of psychological tests derived from such a theory. As we maintained,

although one could argue that the "structural architecture" underlying the Planning and Attention factors is distinct, as indicated by research in neurology and experimental psychology, the correlational evidence presented here suggests that individual differences in planning and attention are related to the same underlying source of variance. (p. 154, emphasis in the original)

In their response, Naglieri and Das (this issue) appear to argue that, because the PASS theory is based on a neuropsychological model and supported by evidence dating back more than 25 years, the lack of congruence between the PASS theory and the test battery reported by Naglieri et al. must be due to imperfections in their initial attempts to operationalize the PASS constructs. This is a reasonable hypothesis, based on these results, but only one of several. 
Naglieri and Das have recently revised the battery of PASS tests presented in Naglieri et al. (1991). In addition, Naglieri, Braden, and Gottling (1993) have recently published confirmatory factor analysis results in support of the superior fit of the PASS model over several alternative models, including a (PA)SS model and a hierarchical model. The article by Naglieri et al. (1993), which was published in September 1993, was not available to us at the time of our reanalyses. Our article was initially submitted for publication in November 1992, and accepted in March 1993. For future research, however, it may be important to note that the results of Naglieri et al. (1993) are based on a revised battery of PASS tests and a sample of much younger children than that used in our study. We reanalyzed data from the Grades 5-12 group in Naglieri et al. (1991), whereas Naglieri et al. (1993) analyzed data from a sample of kindergartners. To the best of our knowledge, data on the factor structure of this more recent battery of PASS tests with a sample of older children have not yet been reported. We encourage independent attempts at replication of our results with this new battery and a sample of children in Grades 5-12.

In addition to these criticisms, Naglieri and Das (this issue) claim that we generalized from the results of our study to the battery of PASS tests in DasNaglieri: Cognitive Assessment System Standardization Edition (DN:CAS) (Das \& Naglieri, 1993). They argue that this is mistaken because the battery of tests in the DN:CAS is substantially different from the test battery reported by Naglieri et al. (1991) that we reanalyzed. Although we wrote that "inclusion of the present battery of planning and attention tests in Das and Naglieri's new measure of mental ability (the Cognitive Assessment System) is suspect," we believe it is clear that the phrase "present battery" referred to the test battery we reanalyzed, not to all future batteries of PASS tasks. Nevertheless, we emphasized this point by warning that

as the results of any factor analysis technique depend upon the specific variables that are entered (see Carroll, 1993), substantial revision of the present battery of tests may lead to different results than those obtained in the present study. (p. 154)

In addition to underscoring generalizability limitations related to test sampling, while discussing the apparent indistinguishability of the Planning and Attention factors we noted generalizability limitations related to subject sampling:

Contrary to the contention of Naglieri et al. (1991) that "planning and attention have emerged as separate factors distinct from simultaneous and successive coding" (p. 431), the results of this study also suggest that planning and attention are not "functionally distinct," as claimed, but functionally indistinguishable, at least with children in Grades 5-12 and with this particular battery of tasks. (emphasis added) 
As a further caution, we stressed the need for replication of our findings by stating that "cross-validation of these results on a different data set is also required before firm conclusions regarding the latent structure of the PASS tests can be made." Regarding the interpretation of our results, we have therefore not "gone too far with too little data," "missed the big picture," or "clouded the issues," as Naglieri and Das conjecture. On the contrary, our interpretation of the data was both reasonable and properly cautious.

\section{CONCLUSION}

In this reply, we have argued that the criticisms of our study by Naglieri and Das (this issue) are based on misinterpretation of our aims and conclusions. Our own conclusions and interpretations, which pertain only to the factor structure of the battery of tests presented in Naglieri et al. (1991), cannot be refuted by their comments. Naglieri and Das conclude that "the article by Kranzler and Weng distracts us from the recognition that intelligence tests need to be modernized and based on a sound theory" (their emphasis). We disagree. Our study, which addressed inadequacies in the article by Naglieri et al. (1991), should not distract anyone from the perfectly obvious fact that the assessment of cognitive abilities should be based on sound theory. Consequently, we believe our research will not be looked on as a distraction, as Naglieri and Das opine, but as part of the integral process of conjecture and refutation in the growth of scientific knowledge on the assessment of human intelligence.

\section{ACKNOWLEDGMENTS}

We thank Drs. James J. Algina, Craig L. Frisby, and Frank Pajares for comments on an earlier version of this manuscript.

\section{REFERENCES}

Brody, N. (1994). Cognitive abilities. Psychological Science, 5, 63-68.

Carroll, J. B. (1993). Human cognitive abilities: A survey of factor-analytic studies. New York: Cambridge University Press.

Das, J. P., \& Naglieri, J. A. (1993). Das-Naglieri: Cognitive assessment system standardization edition. Chicago: Riverside.

Eysenck, H. J. (1994). [Review of Human cognitive abilities: A survey of factor-analytic studies.] Personality and Individual Differences, 16, 199.

Jöreskog, K. G., \& D. Sörbom (1989). LISREL VII: A guide to the program and applications (2nd ed.). Chicago: SPSS Inc.

Lambert, N. M. (1990). Consideration of the Das-Naglieri cognitive assessment system. Journal of Psychoeducational Assessment, 8, 338-343.

Naglieri, J. A., Braden, J. P., \& Gottling, S. H. (1993). Confirmatory factor analysis of the planning, attention, simultaneous, successive (PASS) cognitive processes model for a kindergarten sample. Joumal of Psychoeducational Assessment, 11, 259269. 
Naglieri, J. A., Das, J. P., Stevens, J. J., \& Ledbetter, M. F. (1991). Confirmatory factor analysis of planning, attention, simultaneous, and successive cognitive processing tasks. Joumal of School Psychology, 29, 1-17.

Reschly, D. J., \& Wilson, M. S. (1990). Cognitive processing versus traditional intelligence: Diagnostic utility, intervention implications, and treatment validity. Joumal of School Psychology, 19, 443-458.

Sternberg, R. J. (1994). 468 factor-analyzed data sets: What they tell us and don't tell us about human intelligence. Psychological Science, 5, 63-65.

Telzrow, C. F. (1990). Does PASS pass the test? A critique of the Das-Naglieri cognitive assessment system. Journal of Psychoeducational Assessment, 8, 344-355. 